\title{
COMBINATION OF RANDOM ISOTHERMAL AMPLIFICATION AND NANOPORE SEQUENCING FOR RAPID IDENTIFICATION OF THE CAUSATIVE AGENT OF AN OUTBREAK
}

Sören Hansen ${ }^{1}$, Oumar Faye ${ }^{2}$, Sabri S. Sanabani ${ }^{3}$, Martin Faye, Ousmane Faye ${ }^{2}$, Susanne Böhlken-Fascher ${ }^{1}$, Amadou A. Sall ${ }^{2}$, Manfred Weidmann ${ }^{4}$, Claus-Peter Czerny ${ }^{1}$ and Ahmed Abd El Wahed ${ }^{1}$

${ }^{1}$ Division of Microbiology and Animal Hygiene, University of Goettingen, Goettingen, Germany; '2nstitut Pasteur de Dakar, Dakar, Senegal; '3epartment of Pathology, School of Medicine, University of São Paulo, São Paulo, Brazil; ${ }^{4}$ Institute of Aquaculture, University of Stirling, Stirling, Scotland, UK.

Accepted refereed manuscript of:

Hansen S, Faye O, Sanabani SS, Faye M, Böhlken-Fascher S, Faye O, Sall AA, Bekaert M, Weidmann M, Czerny C \& Abd El Wahed A (2018) Combination Random Isothermal Amplification and Nanopore Sequencing For Rapid Identification of the Causative Agent of an Outbreak, Journal of Clinical Virology, 106, pp. 23-27.

DOI: 10.1016/j.jcv.2018.07.001

(C) 2018, Elsevier. Licensed under the Creative Commons Attribution-

NonCommercial-NoDerivatives 4.0 International

http://creativecommons.org/licenses/by-nc-nd/4.0/ 


\section{Abstract}

2 Introduction: As an outbreak of fever of unknown origin usually starts with

3 nonspecific symptoms and a case definition is only slowly developed and 4 adapted, therefore, identifying the causative agent is crucial to ensure suitable 5 treatment and/or control measures. Polymerase Chain Reaction (PCR) as a gold 6 standard of the molecular diagnostics depends on the previous knowledge of the 7 pathogen genome sequences. Next generation Sequencing is an alternative method, 8 which can be applied to identify the pathogen responsible for the outbreak through 9 sequencing all nucleic acids present in a sample extract. Sequencing data obtained can potentially identify new agents or new variants of known agents.

11 Aim: In this pilot study, we explored a sequencing protocol relying on multiple displacement isothermal amplification and nanopore sequencing in order to allow the identification of the causative agent in a sample. To develop the procedure for use in a suitcase laboratory, a mock sample consisting of supernatant from a Zika virus tissue culture was used.

16 Results: The whole procedure took around eight hours including sample preparation and data analysis using BLAST search. In total, 63,678 sequence files covering around 10,000 bases were extracted. BLAST search revealed the presence of Zika virus, which was close to an isolate from Senegal.

20 Conclusion: In conclusion, the protocol has potential for point of need sequencing to

21 identify RNA viruses. The whole procedure was operated in a suitcase laboratory powered by solar power batteries. However, the procedure is cooling chain dependent and the cost per sequencing run is still very high. In addition, sequencing and data analysis pipelines for optimized and rapid subtraction of background information and assembly of relevant virus information are required. 
27 Identifying the causative agent implicated in an outbreak is crucial for selecting the

28 suitable treatment and/or control measures (1). For example, around 25 pathogens 29 can cause influenza like symptoms in the acute phase and up to 20 pathogens have 30 to be considered for diarrhoea.

31 For direct detection of pathogens, polymerase chain reaction (PCR) is a widely used 32 and well-established test for molecular diagnostics. Since specificity of PCR 33 oligonucleotides depends on known sequences of specific target genes, false 34 negative PCR result might be obtained due to a mismatching sequence of a novel 35 variant of a known pathogen or because of a new emerging infectious agent. An alternative promising technology is next generation Sequencing (NGS), which can be applied to identify the pathogen responsible for the outbreak through sequencing of all nucleic acids in a sample allowing generic detection not limited by specific oligonucleotide design. Additionally, NGS data sets on detected infectious agents can be use for phylogenetic and molecular epidemiological analysis to provide insights on strain and origin of the agent. This information can be crucial for organization and distribution of resources during the outbreak control $(2,3)$.

There are many NGS technologies available such as sequencing by synthesis, using HiSeq and MySeq devices (Illumina, USA). These devices have a high data output, an error rate below $2 \%$ and the possibility to sequence several samples in parallel (4). Nevertheless, there is a high logistic demand through weight, size and costs of

47 the equipment. Furthermore, cumbersome and long sample and library preparation protocols are necessary in order to generate results $(4,5)$. In contrast, nanopore sequencing technology (Oxford Nanopore Technology, UK) uses a pore-protein 
51 electrical conductivity as a DNA molecule passes through the nanopore protein.

52 Recently, Oxford Nanopore Technology developed a pocket sized $(10.5+3.5+2.5$ $\mathrm{cm}$ ) sequencing device (MinION) which has the potential to be applied in the field or rural areas. A flow cell containing the required nanopores is inserted into the MinION in order to operate the sequencing run. The MinION device operates at a constant sequencing temperature $\left(34^{\circ} \mathrm{C}\right)$ and translates the measured changes in current to a real-time nucleotide sequence via USB connection to a laptop (5-8).

Here we describe the establishment of a protocol for rapid identification of RNA viruses combining. random isothermal amplification and nanopore sequencing using Zika virus (ZIKV) as model virus. The protocol was performed in a mobile suitcase laboratory (figure 1) in order to allow implementation in outbreak situation (9).

\section{Materials and Methods}

\section{Sample origin}

ZIKV strains were provided by WHO collaborating Center at the Institute Pasteur of

Dakar in Senegal. The monkey strain MR766 and the human strain HD78788 were isolated in 1947 (in Uganda) and 1991 (in Senegal) in Africa, respectively, during surveillance. Viral stocks were prepared by inoculating viral strains into Aedes pseudoscutellaris clone 61 (AP61) monolayer. Cells were grown in cell culture flasks (25 cm2) until they reached a confluence of approximately $80 \%$. The medium was discarded, and $150 \mu \mathrm{l}$ virus solution was added to the cells. The flasks were gently agitated every 15 min during incubation to enhance viral infection. After $1 \mathrm{~h}, 5 \mathrm{ml}$ of Leibovitz 15 (L-15) growth medium (GibcoBRL, Grand Island, NY, USA)

74 supplemented with $5 \%$ heat-inactivated fetal bovine serum (FBS) (GibcoBRL, Grand 
75 Island, NY, USA), 10\% Tryptose Phosphate $1 \%$ glutamine, $1 \%$ penicillin-

76 streptomycin, 0.05\% amphotericin B [Fungizone] (Sigma, Gmbh, Germany) was

77 added and the infected cells were incubated at $28^{\circ} \mathrm{C}$ without $\mathrm{CO} 2$ until a cytopathic 78 effect was observable.. Viral infection was confirmed by an indirect 79 immunofluorescence assay (IFA) using specific hyper-immune mouse ascitic fluid, as 80 described previously (Digoutte et al., 1992).

\section{Sample preparation}

82 Zika virus (ZIKV) RNA was extracted from cell culture supernatant using the QIAamp 83 Viral RNA Mini Kit (QIAGEN Hilden, Germany) following the manufacturer`s 84 instructions. The RNA quantity was measured by NanoDrop ND-1000 spectrometer 85 (Thermo Scientific, Waltham, MA, USA). For elimination of genomic DNA and reverse 86 transcription, the QuantiTect Reverse Transcription Kit (QIAGEN Hilden, Germany) 87 was employed using a prolonged incubation time $(25 \mathrm{~min})$ for the reverse 88 transcription step. Second strand cDNA Synthesis was performed with the NEBNext 89 mRNA Second Strand Synthesis Module (New England Biolabs, Ipswich, MA, USA). 90 The double-stranded cDNA (ds-cDNA) was purified with the 1.8X Agencourt AMPure 91 XP Beads Kit (Beckman Coulter, Brea, CA, USA), eluted in $55 \mu$ nuclease-free water and quantified (NanoDrop ND-1000). To fragment and increase the amount of DNA, random amplification was done using the REPLI-g UltraFast Mini Kit (QIAGEN

94 Hilden, Germany), Briefly, $1 \mu \mathrm{l}$ of ds-cDNA, containing at least $10 \mathrm{ng}$, was incubated with $1 \mu \mathrm{l}$ denaturation buffer at room temperature. To terminate the denaturation, $2 \mu \mathrm{l}$ neutralization buffer was added after 3 min. The denatured ds-cDNA was mixed with

$97 \quad 16 \mu \mathrm{l}$ of the master mix containing $15 \mu \mathrm{l}$ REPLI-g UltraFast reaction buffer and $1 \mu \mathrm{l}$ 98 REPLI-g UltraFast DNA polymerase and incubated at $30^{\circ} \mathrm{C}$ for $90 \mathrm{~min}$. The reaction 99 mix was heated to $65^{\circ} \mathrm{C}$ for 3 min to inactivate the reaction enzymes. Then, the DNA 
100

101 free water and quantified (NanoDrop ND-1000).

102

103

104

105 114 MinION device.

\section{Library preparation and sequencing}

For library preparation, the protocol for amplicon sequencing, SQK-NSK007, was used as recommended by Oxford Nanopore Technology. Briefly, $45 \mu$ l containing at least $1 \mu \mathrm{g}$ ds-cDNA were used for end-repairing and dA-tailing using the NEBNext Ultra II end-repair / dA-tailing module. The end-prepped DNA was purified with the 1.8X Agencourt AMPure XP Beads Kit and eluted in $31 \mu$ nuclease free water. DNA recovery aim was at least $700 \mathrm{ng} / \mu \mathrm{l}$. Adapter ligation and tethering was carried out with the NEB Blunt/TA Ligase Master Mix. The DNA was purified using the Dynabeads ${ }^{\circledR}$ MyOne ${ }^{\mathrm{TM}}$ Streptavidin C1 Kit (Thermo Fisher Scientific, Waltham, MA, USA) and solved in $25 \mu$ l of Oxford Nanopores` Elution Buffer. Six microliter of the adapted and tethered DNA was mixed with $31.5 \mu \mathrm{l}$ nuclease free water and $37.5 \mu \mathrm{l}$ of Oxford Nanopores` Running Buffer FM1 and then loaded into the flow cells in the

\section{Data processing}

116 The MinION device generates data in fast5 format. These reads were processed with 117 the METRICHORE AGENT (Oxford Nanopore Technology, Oxford, UK). Afterwards,

118 the files were transformed to fastq format with PORETOOLS (10). Duplicate reads 119 were deleted and the remaining sequences were loaded in BLAST search using 120 GENEIOUS 9.1.6 (Biomatters Ltd., Auckland, New Zealand). Contigs were aligned to 121 Zika strain KF383115 via Map to Reference option in GENEIOUS. 
124 The described procedure took around eight hours as shown in table 1. In total, 63678

125 sequences were extracted and transformed to fastq format. After uploading the 126 sequences to BLAST, ZIKV sequences were identified in approximately $4 \%$ of the 127 reads. The complete original ZIKV sequence (GenBank accession number: 128 KF383115) was recovered with 2454 reads with an average read length of XXX 129 (Max.: 585, Min.: 36, Std. Dev.: 122.6) (figure 2 and 3). The average coverage was $x$ 130 fold the minimum coverage was $x$-fold. Pairwise identity in BLAST analysis was $13167.4 \%$.

132 Additionally a total of 411 correct ZIKV reads were found in the FAST5 fail sequence 133 file. If included in the assembly they matched correctly to the respective ZIKV 134 sequence (figure 3).

136 Table 1: Sequencing Workflow

\begin{tabular}{|c|c|c|}
\hline Procedure & Reagents/Software & $\begin{array}{c}\text { Time } \\
(\mathbf{m i n})\end{array}$ \\
\hline RNA extraction & QIAamp Viral RNA Mini Kit & 30 \\
\hline $\begin{array}{c}\text { DNA digestion and reverse } \\
\text { transcription }\end{array}$ & QuantiTect Reverse Transcription Kit & 35 \\
\hline $\begin{array}{c}\text { second strand cDNA synthesis } \\
\text { NEBNext mRNA Second Strand } \\
\text { Synthesis Module }\end{array}$ & 90 \\
\hline random isothermal amplification & REPLI-g UltraFast Mini Kit & 120 \\
\hline library preparation & $\begin{array}{c}\text { Nanopore sequencing kits: SQK- } \\
\text { NSK007 }\end{array}$ & 70 \\
\hline sequencing & MinlON device and R9 flow cell & 20 \\
\hline data analysis and BLAST search & PORETOOLS and Geneious 9.1.6 & 120 \\
\hline Total & & 485 \\
\hline
\end{tabular}




\section{Discussion}

140 Identifying the causative agent of an outbreak using sequencing instead of molecular

141 techniques like PCR could have a high impact on selecting and implementing the 142 right patient management and control measures.

143 The most widespread sequencing device is the MiSeq, as Illumina`s smallest device, 144 which has still a size of $68.6+52.3+56.5 \mathrm{~cm}$ and a weight of approximately $57 \mathrm{~kg}$. 145 Moreover, it has a higher data output (15 Giga bases) in comparison to the MinION 146 (10 Giga bases). Nevertheless, read length by MiSeq is limited to around 300 bp and 147 a maximum of $22-25$ million reads can be produced in a run time between $4 \mathrm{~h}$ and $14856 \mathrm{~h}(11,12)$. In contrast, the MinION has through its nanopore technology no limit in 149 read length and number. Moreover, reads are generated in 20-120 min and data are 150 easily accessible on laptop or PC.

151 We have discovered that $1 / 5$ of the correct ZIKV reads was placed into the "fail" file.

152 The METRICHORE AGENT classifies the reads into pass and fail reads by neuronal 153 network computing assessing definite conductivity readout events at the pore exit for 154 5-6 mers. This complicated sequence definition needs quality scoring to decide on 155 the statistical trustworthiness of the sequencing result. Fails are defined through the 156 following approach. Initially base calling (1D base calling) of template and 157 complement reads is performed separately. If the resulting sequence length ratio is 158 between 0.5-2.0, all sequences are stacked together for base 2D base calling. If 159 resultant 2D sequences are assessed with a Q-score $>9$ they are sorted into a 160 FAST5 fail sequence file (13).

161 Short Illumina device reads have a $0.1 \%$ non-random error rate, which means an 162 error at one site can still dominate the base calling process. The MinION reads have a $10 \%$ error rate but sites are distributed at random throughout the sequence which 
164 is compensated for by base calling and which therefore do not dominate at one site

165 reducing the overall error rate in comparison to Illumina reads (14). Our results 166 suggest that the analysis algorithm and the Q-score need to be optimised for viral 167 RNA sequences. At this current development stage therefore a recommended 168 assembly approach would be first to use all pass reads to identify the infectious 169 agent. To improve the result, the fail reads can be included in a $2^{\text {nd }}$ step.

170 In general however the passed sequence assembly result already produce a robust 171 result with a average coverage of 40.

172 The MinION was successfully used in the Ebola virus outbreak in Guinea (7) and 173 during the Zika virus outbreak in Brazil (15). In both cases, specific PCR fragment 174 sequencing strategies were used. RT-PCR assays were applied to reverse transcribe 175 RNA and create multiple fragments to increase the sequencing efficacy $(7,16)$. This 176 strategy limits sequencing output to targeted agents, which is ideal for molecular 177 epidemiological analysis. The use of PCR leads to logistic issues due to heavy 178 devices and requirement of a cold chain for the reagents. In Brazil, this was solved 179 by transporting the whole laboratory in a caravan. The generic sequencing approach 180 described here is intended for diagnostic identification of unknown infectious agents. 181 It uses only random isothermal steps throughout the procedure and PCR cycling is 182 not required which avoids the use of a thermal cycler.

183 We have already shown that isothermal amplification can be easily implemented in a 184 mobile suitcase laboratory $(9,17,18)$ and we successfully adapted this concept for 185 the workflow needed for library preparation for the MinION sequencing procedure 186 (figure 1). The suitcase, contains all materials and reagents needed for sequencing in 187 one box of $56.0+45.5+26.5 \mathrm{~cm}$ in size and less than $23 \mathrm{~kg}$ in weight. 
188 All steps of data collection and analysis except the BLAST search were performed 189 offline using MINKNOW and METRICHORE AGENT as well as GENEIOUS. This is a 190 major improvement since during the Ebola outbreak base calling for MinION datasets 191 was only possible through cloud computing which needed internet capacity often not 192 available locally (7). The simple structure and clear layout of these analysis 193 programmes makes it easy for users without bioinformatic background to obtain 194 basic information about origin and phylogeny of the sequenced target. Therefore, a 195 bioinformatician is not necessarily needed for analysis of the datasets obtained. To perform BLAST offline a database of infectious agent sequences only located on the 197 laptop needs to be assembled. It could be replenished with new entries to GenBank 198 whenever online.

199 Currently, the following challenges have to be solved. In our hands, the sequencing 200 reagents can be kept at $25^{\circ} \mathrm{C}$ for one day without any changes in their efficacy 201 (confirmed by Oxford Nanopore Technologies, UK). However for long-term storage a $202-20^{\circ} \mathrm{C}$ freezer is still required. Moreover, the price per sequencing run is very high 203 (around $\$ 1500$ ), as one flow cell costs between $\$ 500$ and $\$ 900$ depending on the 204 amount of ordered flow cells. In addition, the shelf life of the flow cells is around 8 205 weeks at $4^{\circ} \mathrm{C}$. One of the biggest drawbacks is that the manufacturer is progressively 206 changing the reagents and flow cells so that it is difficult to match biochemistry to 207 flow cells.

208 The goal of this pilot study was to establish a protocol for pathogen identification 209 during an outbreak field investigation. In principle this seems possible in a suitcase 210 laboratory setup. The next steps will be to assemble an offline solution to compare 211 identified sequences with preloaded database and to identify cold chain independent 212 reagents. 
1. Ni PX, Ding X, Zhang YX, Yao X, Sun RX, Wang P, Gong YP, Zhou JL, Li DF, Wu HL, Yi X, Yang L, Long Y. 2015. Rapid detection and identification of infectious pathogens based on high-throughput sequencing. Chin Med $\mathrm{J}$ (Engl) 128:877-883.

2. Robinson ER, Walker TM, Pallen MJ. 2013. Genomics and outbreak investigation: from sequence to consequence. Genome Med 5:36.

3. Croucher NJ, Didelot X. 2015. The application of genomics to tracing bacterial pathogen transmission. Curr Opin Microbiol 23:62-67.

4. Liu L, Li Y, Li S, Hu N, He Y, Pong R, Lin D, Lu L, Law M. 2012. Comparison of next-generation sequencing systems. J Biomed Biotechnol 2012:251364.

5. Hoenen T, Groseth A, Rosenke K, Fischer RJ, Hoenen A, Judson SD, Martellaro C, Falzarano D, Marzi A, Squires RB, Wollenberg KR, de Wit E, Prescott J, Safronetz D, van Doremalen N, Bushmaker T, Feldmann F, McNally K, Bolay FK, Fields B, Sealy T, Rayfield M, Nichol ST, Zoon KC, Massaquoi M, Munster VJ, Feldmann H. 2016. Nanopore Sequencing as a Rapidly Deployable Ebola Outbreak Tool. Emerg Infect Dis 22:331-334.

6. Cherf GM, Lieberman KR, Rashid H, Lam CE, Karplus K, Akeson M. 2012. Automated forward and reverse ratcheting of DNA in a nanopore at 5-A precision. Nat Biotechnol 30:344-348.

7. Quick J, Loman NJ, Duraffour S, Simpson JT, Severi E, Cowley L, Bore JA, Koundouno R, Dudas G, Mikhail A, Ouedraogo N, Afrough B, Bah A, Baum JH, Becker-Ziaja B, Boettcher JP, Cabeza-Cabrerizo M, CaminoSanchez A, Carter LL, Doerrbecker J, Enkirch T, Garcia-Dorival I, Hetzelt N, Hinzmann J, Holm T, Kafetzopoulou LE, Koropogui M, Kosgey A, Kuisma E, Logue CH, Mazzarelli A, Meisel S, Mertens M, Michel J, Ngabo D, Nitzsche K, Pallasch E, Patrono LV, Portmann J, Repits JG, Rickett NY, Sachse A, Singethan K, Vitoriano I, Yemanaberhan RL, Zekeng EG, Racine T, Bello A, Sall AA, Faye O, et al. 2016. Real-time, portable genome sequencing for Ebola surveillance. Nature 530:228-232.

8. Laver T, Harrison J, O'Neill PA, Moore K, Farbos A, Paszkiewicz K, Studholme DJ. 2015. Assessing the performance of the Oxford Nanopore Technologies MinION. Biomol Detect Quantif 3:1-8.

9. Abd El Wahed A, Weidmann M, Hufert FT. 2015. Diagnostics-in-a-Suitcase: Development of a portable and rapid assay for the detection of the emerging avian influenza A (H7N9) virus. J Clin Virol 69:16-21.

10. Loman NJ, Quinlan AR. 2014. Poretools: a toolkit for analyzing nanopore sequence data. Bioinformatics 30:3399-3401.

11. Urban JM, Bliss, Jacob, Lawrence, Charles E, Gerbi, Susan A 2015. Sequencing ultra-long DNA molecules with the Oxford Nanopore MinION. BioRxiv doi:10.1101/019281.

12. Junemann S, Sedlazeck FJ, Prior K, Albersmeier A, John U, Kalinowski J, Mellmann A, Goesmann A, von Haeseler A, Stoye J, Harmsen D. 2013. 
Updating benchtop sequencing performance comparison. Nat Biotechnol 31:294-296.

1. Digoutte JP, Calvo-Wilson MA, Mondo M, Traore-Lamizana M, Adam F.

286 Continuous cell lines and immune ascitic fluid pools in arbovirus detection. Res Virol.

287 1992, vol. 143: 417-422.

13. Lu H, Giordano F, Ning Z. 2016. Oxford Nanopore MinION Sequencing and Genome Assembly. Genomics Proteomics Bioinformatics 14:265-279.

14. Goodwin S, McPherson JD, McCombie WR. 2016. Coming of age: ten years of next-generation sequencing technologies. Nat Rev Genet 17:333-351.

15. Quick J, Grubaugh ND, Pullan ST, Claro IM, Smith AD, Gangavarapu K, Oliveira G, Robles-Sikisaka R, Rogers TF, Beutler NA, Burton DR, LewisXimenez LL, de Jesus JG, Giovanetti M, Hill SC, Black A, Bedford T, Carroll MW, Nunes M, Alcantara LC, Jr., Sabino EC, Baylis SA, Faria NR, Loose M, Simpson JT, Pybus OG, Andersen KG, Loman NJ. 2017. Multiplex PCR method for MinION and Illumina sequencing of Zika and other virus genomes directly from clinical samples. Nat Protoc 12:1261-1276.

16. Faria NR, Sabino EC, Nunes MR, Alcantara LC, Loman NJ, Pybus OG. 2016. Mobile real-time surveillance of Zika virus in Brazil. Genome Med 8:97.

17. Faye O, Faye O, Soropogui B, Patel P, El Wahed AA, Loucoubar C, Fall G, Kiory D, Magassouba N, Keita S, Konde MK, Diallo AA, Koivogui L, Karlberg H, Mirazimi A, Nentwich O, Piepenburg O, Niedrig M, Weidmann M, Sall AA. 2015. Development and deployment of a rapid recombinase polymerase amplification Ebola virus detection assay in Guinea in 2015. Euro Surveill 20.

18. Mondal D, Ghosh P, Khan MA, Hossain F, Bohlken-Fascher S, Matlashewski G, Kroeger A, Olliaro P, Abd El Wahed A. 2016. Mobile suitcase laboratory for rapid detection of Leishmania donovani using recombinase polymerase amplification assay. Parasit Vectors 9:281. 